\title{
Pulmonary artery myxoma as a rare cause of dyspnea for a young female patient
}

\author{
Shu-Chien Huang, MD, ${ }^{a, b}$ Meng-Lin Lee, MD, ${ }^{a}$ Shyh-Jye Chen, MD, ${ }^{c}$ Mu-Zon Wu, MD, ${ }^{d}$ \\ and Chung-I Chang, MD, ${ }^{a}$ Taipei, Taiwan
}

$\mathrm{F}$ or a patient featuring a filling defect in the pulmonary artery, pulmonary emboli is typically cited as the most frequent diagnosis; however, although typically rare, a tumor in the pulmonary artery should be included in the differential diagnosis. ${ }^{1}$ Herein we report on a patient for whom a primary myxoma originated in the left pulmonary artery and discuss the possible hint to make the differential diagnosis.

\section{Clinical Summary}

A 32-year-old, nonsmoking female patient had been complaining of progressive dyspnea for 3 years. This patient revealed mild chest tightness and palpitations but no evidence of syncope or hemoptysis, and she did not reveal cyanosis, edema, or hepatomegaly. The patient did not take any form of oral contraceptives and featured no history of leg swelling. Right ventricular heave, a grade II/VI systolic murmur over the patient's left upper sternal border, and a split-second heart sound were noted on physical examination. The patient's chest radiograph revealed prominent pulmonary conus without evidence of cardiomegaly (Figure 1, A). The patient's electrocardiogram revealed sinus tachycardia and right ventricular hypertrophy, after which echocardiography was arranged, which revealed flow acceleration in the right pulmonary artery and virtually no flow in the left pulmonary artery. Under the impression of an obstructed pulmonary artery, a computed tomographic (CT) scan was performed, revealing irregular filling defects in the pulmonary trunk extending bilaterally into both the left and right pulmonary arteries (Figure 1,B). The vascular duplex did not reveal any evidence of thrombosis of the leg veins. The blood coagulation profile, abdominal CT scan, and serum levels of $\alpha$ fetoprotein, carcinoembryonic antigen, and carbohydrate antigen 19-9 all were within normal ranges. A perfusion scan was performed, demonstrating a virtual absence of left-lung perfusion, which suggested a diagnosis of a primary tumor in the left pulmonary artery.

Surgical intervention was then performed for this patient, as was arteriotomy of the main pulmonary artery, which revealed a $5 \times 3 \times 3-\mathrm{cm}$, soft, gelatinous, phylloid, friable mass (Figure 2, A)

From the Departments of Surgery ${ }^{\mathrm{a}}$, Traumatology ${ }^{\mathrm{b}}$, Radiology ${ }^{\mathrm{c}}$, and Pathology, ${ }^{\mathrm{d}}$ National Taiwan University Hospital, Taipei, Taiwan.

Received for publication Nov 24, 2005; accepted for publication Dec 30, 2005.

Address for reprints: Chung-I Chang, MD, Department of Surgery, National Taiwan University Hospital, 7 Chung-Shan S Rd, Taipei 100, Taiwan (E-mail: joey@ha.mc.ntu.edu.tw).

J Thorac Cardiovasc Surg 2006;131:1179-80

$0022-5223 / \$ 32.00$

Copyright (C) 2006 by The American Association for Thoracic Surgery

doi:10.1016/j.jtcvs.2005.12.052

The tumor featured a sessile base located within the left pulmonary artery proximate to the ligamentum arteriosus (Figure 2, B). After complete resection of the tumor, with the stalk base being entirely removed, the remaining defect in the pulmonary artery was repaired. No evidence of any tumor was found in the distal pulmonary arteries, right ventricle, or right atrium. The pathologic examination of the removed tissue revealed a typical myxoma. The patient recovered well, and follow-up CT revealed patent pulmonary arteries.

\section{Discussion}

Primary tumors of the pulmonary artery are quite rare, and most reported cases are sarcomas. ${ }^{1}$ Although a myxoma is the most common primary cardiac tumor for human subjects, as revealed by a thorough Medline search, to the best of our knowledge, only 2 cases of primary myxoma arising in the pulmonary artery have been reported in the literature, and only one of them was in English., 2,3

The filling defect in the pulmonary tree should be differentiated from a diagnosis of pulmonary emboli because the treatments for the 2 diagnoses differ markedly. It has been reported that patients were treated with anticoagulation, thrombolysis, or both before a diagnosis of pulmonary artery tumor was made. ${ }^{1,3}$ As regards our patient, the young lady did not reveal any risk factors for pulmonary emboli, such as venous thrombosis, immobilization, hemoptysis, malignancy, or hypercoagulability, and thus the likelihood of pulmonary emboli was quite low.

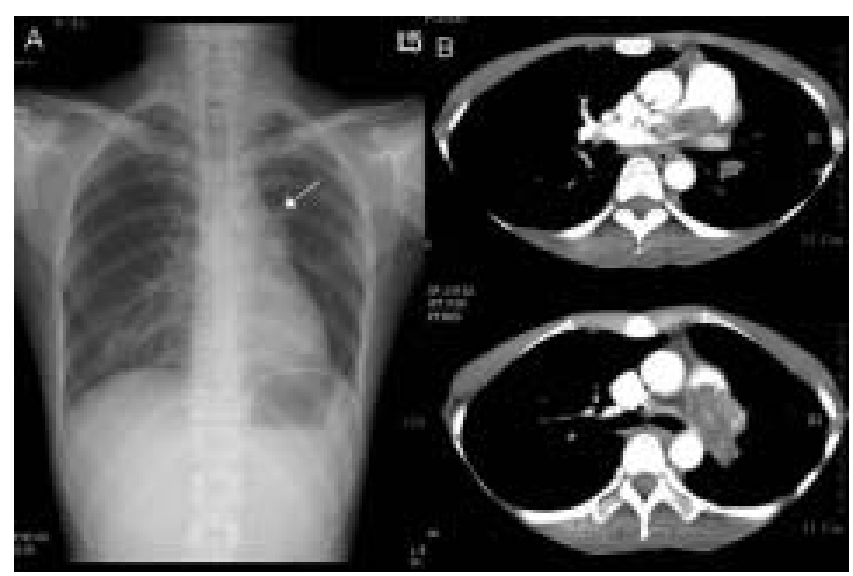

Figure 1. Preoperative images. A, The patient's chest radiograph revealed prominent pulmonary conus. $B$, Computed tomography revealed evidence of an irregular filling defect existing from the distal pulmonary trunk to both pulmonary arteries. 

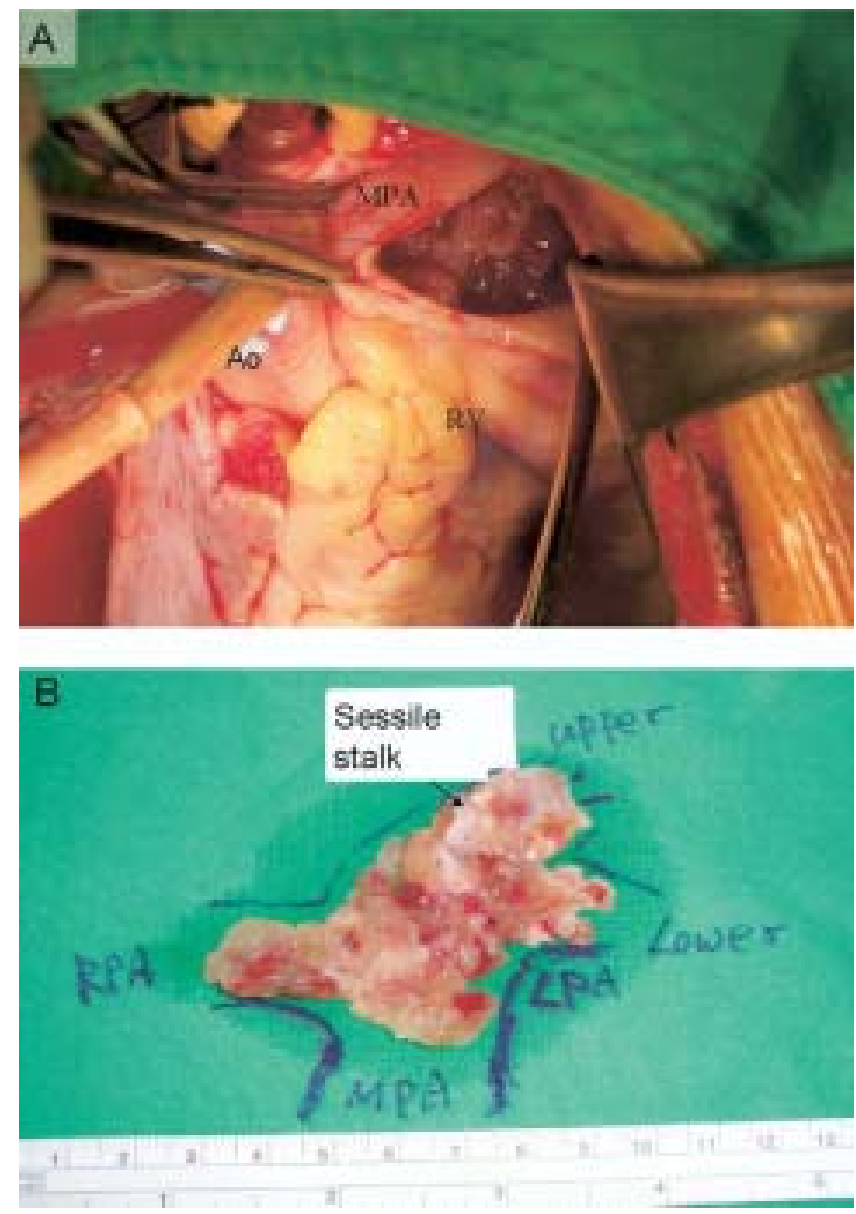

Figure 2. A, Intraoperative photography revealed the myxoma located within the opened pulmonary artery. MPA, Main pulmonary artery; Ao, aorta; RV, right ventricle. B, The surgical specimen can be seen after complete resection. $R P A$, Right pulmonary artery; $M P A$, main pulmonary artery; $L P A$, left pulmonary artery.
By means of a thorough review of the imagery results for our patient, as also presented in the 2003 report of Huang and colleagues, ${ }^{3}$ the irregular filling defect present in the pulmonary artery appeared similar to a fungating mass with irregular surfaces and not similar to a typical example of pulmonary emboli, which features a sharp interface with intravascular contrast material. ${ }^{4} \mathrm{We}$ suggest a high index of suspicion of a tumor if the history or the imagery do not resemble those of typical pulmonary emboli.

The treatment of choice for a pulmonary artery tumor features surgical resection, but for patients with sarcomas of the pulmonary artery, recurrence is common, and patient survival is relatively poor. For patients with a primary myxoma located in the pulmonary artery, the long-term results would appear to not be well known. For the only case that we were able to find reported in the relevant English-language literature, ${ }^{3}$ the patient died after surgical intervention as a result of right ventricular failure. Although a good result is generally expected after resection of cardiac myxomas, the recurrence of primary myxomas has been reported in the literature. ${ }^{5}$ We advise that such patients be regularly followed up.

\section{References}

1. Choong CK, Lawton JS, Moon MR, et al. Failure of medical therapy for pulmonary "thromboembolic" disease: beware the unsuspected primary sarcoma of the pulmonary artery. J Thorac Cardiovasc Surg. 2004;128: 763-5.

2. Makoeva LD, Pochechueva GA, Moskaleva MI, et al. [A rare case of myxoma of the pulmonary artery trunk]. Klin Med (Mosk). 1991;69: 74-5.

3. Huang $\mathrm{CY}$, Huang $\mathrm{CH}$, Yang $\mathrm{AH}$, et al. Solitary pulmonary artery myxoma manifesting as pulmonary embolism and subacute cor pulmonale. Am J Med. 2003;115:680-1.

4. Wittram C, Maher MM, Yoo AJ, et al. CT angiography of pulmonary embolism: diagnostic criteria and causes of misdiagnosis. Radiograph ics. 2004;24:1219-38.

5. Shinfeld A, Katsumata T, Westaby S. Recurrent cardiac myxoma: seeding or multifocal disease? Ann Thorac Surg. 1998;66:285-8. 